\title{
Galactic sources of high energy neutrinos
}

\author{
Felix Aharonian \\ Dublin Institute for Advanced Studies, 5 Merrion Square, Dublin 2, Ireland \& \\ Max Planck Institut für Kernphysik, Saupfercheckweg 1, 69117 Heidelberg, Germany \\ E-mail: felix.aharonian@mpi-hd.mpg.de
}

\begin{abstract}
The undisputed galactic origin of cosmic rays at energies below the so-called knee around $10^{15} \mathrm{eV}$ implies an existence of a nonthemal population of galactic objects which effectively accelerate protons and nuclei to TeV-PeV energies. The distinct signatures of these cosmic accelerators are high energy neutrinos and $\gamma$-rays produced through hadronic interactions. While $\gamma$-rays can be produced also by directly accelerated electrons, high energy neutrinos provide the most straightforward and unambiguous information about the nucleonic component of accelerated particles. The planned $\mathrm{km}^{3}$-volume class high energy neutrino detectors are expected to be sensitive enough to provide the first astrophysically meaningful probes of potential VHE neutrino sources. This optimistic prediction is based on the recent discovery of high energy $\gamma$-ray sources with hard energy spectra extending to $10 \mathrm{TeV}$ and beyond. Amongst the best-bet candidates are two young shell-type supernova remnants - RXJ 1713.74946 and RXJ 0852.0-4622, and perhaps also two prominent plerions - the Crab Nebula and Vela X. Because of strong absorption of $\mathrm{TeV} \gamma$-rays, one may expect detectable neutrino fluxes also from (somewhat fainter) compact $\mathrm{TeV} \gamma$-ray emitters like the binary systems LS 5039 and LS I+61 303, and, hopefully, from hypothetical "hidden" or "orphan" neutrino sources.
\end{abstract}

\section{Introduction}

Very High Energy ( $E \geq 0.1 \mathrm{TeV}$; VHE) neutrinos are unique messengers of nonthermal phenomena in the Universe related to the hadronic interactions of protons and nuclei in cosmic TeVatrons and PeVatrons - Nature's masterly designed machines accelerating particles to TeV and $\mathrm{PeV}$ energies. In this regard VHE neutrinos are complementary to $\gamma$-rays which are produced both in electromagnetic and hadronic interactions. On the other hand, unlike $\gamma$-rays, neutrinos are not fragile; they interact only weakly with the ambient medium - gas, radiation and magnetic fields, and thus carry information about high energy processes occurring in "hidden" regions where the particle accelerators could be located. This concerns, first of all, the regions associated with compact objects - black holes, pulsars, the initial epochs of supernovae explosions, etc. The penetrating potential of neutrinos is important not only for extremely dense environments in which $\gamma$-rays are dramatically absorbed, but also moderately opaque sources from which we can see $\gamma$-rays, but after significant distortion due to internal and external absorption.

Ironically, this nice (from an astrophysical point of view) feature of neutrinos makes, at the same time, their detection extremely difficult. This explains why over several decades high energy neutrino astronomy has remained essentially a theoretical discipline with many exciting ideas and predictions but without the detection of a single VHE neutrinos source. However, it is expected that, with arrival of the $\mathrm{km}^{3}$-volume class scale detectors like IceCube and KM3NeT (see e.g. refs. [1, 2]), the status of the field will be changed dramatically. Generally, predictions 
of VHE neutrino fluxes from astrophysical objects rely on assumptions with not well-constrained model parameters and, therefore, often contain large (orders of magnitude!) uncertainties. This leaves a significant freedom in speculations on the "best-bet neutrino sources", and consequently allows a broad spectrum of opinions concerning the prospects for detecting the first astrophysical neutrinos - from very enthusiastic statements to rather careful predictions prevailed by a healthy scepticism (see e.g. ref. [3]).

Presently extragalactic objects like Active Galactic Nuclei (AGN) and sources of Gamma Ray Bursts (GRBs) are believed to be the most likely objects to be detected as neutrino sources, and therefore the driving motivation of experimental VHE neutrino astronomy (see e.g. ref. [4]). The current models of AGN and GRBs indeed contain many attractive components (concerning the conditions of particle acceleration and their interactions; see e.g. ref. [5]) that makes these objects potentially detectable sources of VHE neutrinos (see e.g. refs. 6, 7]). On the other hand, the poor understanding of certain aspects of the physics of AGN and especially GRBs, as well as the lack of constraints on neutrino productions rates from $\gamma$-ray observations (because of intrinsic and intergalactic absorption of VHE $\gamma$-rays), formally allow calculations in extreme model-parameter segments which often lead to rather high (in the case of Blazars overoptimistic) neutrino flux predictions.

In contrast to extragalactic objects, the models of potential galactic neutrino sources, in particular the shell type Supernova Remnants (SNRs), Pulsar Wind Nebulae (PWNe), Star Formations Regions and the dense molecular clouds related to them, are robustly constrained by $\gamma$-ray observations of the galactic disk in very-high $(\geq 1 \mathrm{TeV})[8,9$, and ultra-high energy $(\geq 100 \mathrm{TeV})[10$ domains. Typically, the expected fluxes from these objects are below the detection threshold of the planned neutrino detectors. However, the recent HESS discoveries of several TeV $\gamma$-ray sources at the flux level of " 1 Crab", which can be interpreted within the hadronic models of gamma-ray emission, sustain a hope that first $\mathrm{TeV}$ galactic sources will be detected in foreseeable future by $\mathrm{km}^{3}$-volume class instruments like IceCube and Km3NeT.

\section{On the detectability of galactic VHE neutrino sources}

The recent performace studies of the $\mathrm{km}^{3}$-volume scale detectors show that the detection of persistent point-like neutrino sources (for a typical angular resolution of VHE neutrino detectors the "point-like" source implies an object of angular size $\leq 1^{\circ}$ ) for a realistic exposure time (typically, a few years continuous observations) is limited by a flux $F(\geq 1 \mathrm{TeV}) \approx 10^{-11} \nu / \mathrm{cm}^{2} \mathrm{~s}$ (see e.g. refs. [11, 12, 13, 14]). The corresponding energy flux is $f_{\mathrm{E}} \approx 10^{-10} \mathrm{erg} / \mathrm{cm}^{2} \mathrm{~s}$ or somewhat less, depending on the spectrum in the most relevant energy interval between 1 and $100 \mathrm{TeV}$. This exceeds, by two orders of magnitude, the minimum $\gamma$-ray flux detectable in the same energy band. On the other hand, the sensitivity of the $\mathrm{km}^{3}$-scale detectors is comparable or better than the minimum detectable energy flux achieved by the Compton Gamma Ray Observatory detectors (COMPTEL, EGRET) in the $\mathrm{MeV} / \mathrm{GeV} \gamma$-ray band. For an isotropic VHE source located at a distance $d$, the luminosity of $\mathrm{TeV}$ neutrinos can be probed at the level

$$
L_{\nu} \simeq 10^{34}(d / 1 \mathrm{kpc})^{2} \mathrm{erg} / \mathrm{s} .
$$

At first glance, this is a quite modest luminosity, at least for a powerful hadronic source located in a dense environment. Indeed, for production of $\mathrm{TeV}$ neutrinos in $p$ - $p$ interactions with ambient gas of density $n_{0}=n / 1 \mathrm{~cm}^{-3}$, the required total energy in multi- $\mathrm{TeV}$ protons is estimated $W_{p} \simeq t_{\mathrm{pp}} c_{\mathrm{p} \rightarrow \nu}^{-1} L_{\nu} \simeq 10^{49} n_{0}^{-1} d_{\mathrm{kpc}}^{2}$ erg, where $t_{\mathrm{pp}} \approx 10^{15} n_{0}^{-1} \mathrm{~s}$ is the radiative cooling time of protons due to inelastic $p-p$ interactions, and $c_{\mathrm{p} \rightarrow \nu} \approx 0.1$ is the fraction of average energy of a proton transferred to neutrinos. One may conclude that even in a relatively low density environment $\left(\mathrm{n}_{0} \sim 1\right)$ the required total energy can be readily produced in young SNRs through diffusive shock acceleration (see e.g. ref. 15]) or by a powerful pulsar assuming that a major fraction of the spin-down luminosity of the pulsar is converted to an ion-dominated wind (see 
e.g. ref. [16]). However, this kind of estimates can be misleading since they are based on a silent assumption that all particles accelerated during the life-time of the source are effectively confined in a relatively compact region inside or nearby the accelerator. In fact, production of $\mathrm{TeV}$ neutrinos requires protons with energies well beyond $10 \mathrm{TeV}$ the escape of which from the source is difficult to prevent. This, of course, would lead to a significant reduction of the neutrino production efficiency which in the case of $p$ - $p$ interactions can be expressed in the following form: $\eta=L_{\nu} / \dot{W}_{\mathrm{p}}=\min \left[1, t_{\mathrm{esc}} / t_{\mathrm{pp}}\right] \times c_{\mathrm{p} \rightarrow \nu}$, where $t_{\mathrm{esc}}$ is the escape time of nonthermal particles. If the escape proceeds in the diffusion regime, then $t_{\mathrm{esc}}=R^{2} / 2 D(E)$. It is convenient to write the diffusion coefficient $D(E)$ in the following form $D(E)=\xi r_{\mathrm{L}} c / 3=3.3 \times 10^{22} \xi E_{\mathrm{TeV}} B_{\mathrm{mG}}^{-1} \mathrm{~cm}^{2} / \mathrm{s}$, where $E_{\mathrm{TeV}}=E / 1 \mathrm{TeV}$ is the proton energy normalized to $1 \mathrm{TeV}$, and $B_{\mathrm{mG}}=B / 10^{-3} \mathrm{G}$ is the magnetic field in units of $\mathrm{mG}$. Generally the parameter $\xi \geq 1$ is a function of energy. In the most effective confinement regime corresponding to the Bohm diffusion, $\xi=1$. Thus, in a source of a linear size $R_{\mathrm{pc}}=R / 1 \mathrm{pc}$, the production efficiency of $\mathrm{TeV}$ neutrinos, assuming that a hard energy spectrum of protons extends effectively to $100 \mathrm{TeV}$, is

$$
\eta \approx 10^{-2} c_{p \rightarrow \nu} \xi^{-1} R_{\mathrm{pc}}^{2} n_{0} B_{\mathrm{mG}} .
$$

The maximum possible efficiency of a $\mathrm{TeV}$ neutrino source, $\eta \rightarrow c_{p \rightarrow \nu} \approx 0.1$ can in principle be achieved if $R_{\mathrm{pc}}^{2} n_{0} B_{\mathrm{mG}} \geq 10^{2} \xi$. Such a condition can be best fulfilled in objects like giant molecular clouds, with a size $R \sim 10 \mathrm{pc}$, mass $10^{5} M_{\odot}$, and magnetic field $B \geq 0.1 \mathrm{mG}$, provided that the propagation of multi- $\mathrm{TeV}$ protons proceeds close the Bohm diffusion regime. However, Bohm diffusion hardly can be realized in molecular clouds, thus $\eta \ll c_{p \rightarrow \nu}$, typically $\eta \leq 10^{-3}$, which implies that the acceleration power should exceed $\dot{W}_{\mathrm{p}}=\eta L_{\nu} \geq 10^{37} d_{\mathrm{kpc}}^{2} \mathrm{erg} / \mathrm{s}$. This significantly reduces the number of potentially detectable neutrino sources to the most powerful representatives of nonthermal source populations in our Galaxy. In addition to young SNRs and PWNe, possible emitters of $\mathrm{TeV}$ neutrinos are compact binary systems in which the compact object (a black hole or a pulsar) plays the role of particle accelerator, and the dense gas regions, e.g. the atmosphere of the companion star [17, 18, 19, 20] or the accretion plasma around the compact object [21, play the role of the target.

Moreover, in binary systems containing a luminous optical star and a compact object, the photomeson interactions could provide an additional channel for neutrino production, provided that protons are accelerated to energies exceeding the interaction threshold, $E_{\mathrm{th}} \approx$ $(200 \mathrm{MeV} / 3 \mathrm{kT}) \mathrm{m}_{\mathrm{p}} \mathrm{c}^{2} \approx 10^{4} \mathrm{TeV}$ in the case of interactions with the starlight, and three orders of magnitute less for interactions with photons of the accretion disk. For a photon field with a thermal (Planckian) distribution, the interaction and escape times of protons are $t_{\mathrm{esc}} \approx 10^{10} \xi^{-1} R_{\mathrm{pc}}^{2} B_{\mathrm{mG}}(k T / 3 \mathrm{eV}) \mathrm{s}$, and $t_{\mathrm{p} \gamma} \approx 10^{18} L_{37} R_{\mathrm{pc}}^{2}(k T / 3 \mathrm{eV}) \mathrm{s}$, respectively. It is remarkable that both timescales are proportional, although for completely different reasons (!), to the product $R^{2} k T$, therefore the neutrino production efficiency appears independent, for a given luminosity of thermal radiation, of both the source size and the temperature of radiation, but strongly depends on the magnetic field

$$
\eta \approx 10^{-9} \xi^{-1} L_{37} B_{\mathrm{mG}} .
$$

Thus we arrive at the conclusion that the neutrino production via photomeson interactions can be effective only in compact objects with strong magnetic fields $(B \geq 10 \mathrm{kG})$ and high turbulence (for confinement of protons with $\xi \sim 1$ ). The immediate proximity of the luminous star (i.e. its photosphere) or the compact object, e.g the accretion disk or the base of the jet [22, 23, 25], can be sites where the neutrino production proceeds with a reasonably high efficiency.

3. Detectability of neutrino sources in the context of multiwavelength observations The high efficiency of neutrino production is a key condition for the detectability of potential VHE neutrino sources given the limited budget of available energy and the limitted sensitivity of 
detectors. The above qualitative estimates show that this condition can be achieved only with certain combinations of a few key model parameters. Therefore, a careful inspection of these conditions before any detailed numerical calculations is highly advisable.

Independent constraints on the detectability of a VHE neutrino source can be obtained also from analysis of multi-wavelength observations, in particular in the most relevant VHE $\gamma$-ray band. Indeed, $\mathrm{TeV} \gamma$-ray fluxes can be safely used as upper limits for neutrino fluxes, provided, of course, that the internal and external absorption of $\gamma$-rays is negligible. Generally, the production of VHE neutrinos is accompanied by production of $\gamma$-rays, but not vice versa; $\gamma$-rays are copiously produced also in electromagnetic interaction both by electrons (through bremsstrahlung, inverse Compton scattering) and protons (e.g. through the synchrotron and curvature radiations). Moreover, since the main channels of the neutrino and "hadronic" gamma-ray production are decays of charged $\pi^{ \pm}$and neutral $\pi^{0}$ mesons, with decay time of charged pions significantly longer than the decay time of neutral pions, at certain conditions the production of neutrinos can be suppressed compared to $\gamma$-ray production. Indeed, in the lab frame, the decay time of charged pions responsible for $\mathrm{TeV}$ neutrino production is $t_{\pi^{ \pm}}=\left(E_{\pi} / m_{\pi} c^{2}\right) \tau_{\pi^{ \pm}} \approx 2.5 \times 10^{-3}\left(E_{\pi} / 10 \mathrm{TeV}\right) \mathrm{s}$. On the other hand, the cooling time of pions due to inelastic $\pi p$ and $\pi \gamma$ interactions depends on densities of the ambient gas $n_{p}$ and X-ray photons $n_{\mathrm{x}}: t_{\pi p} \sim 10^{14}\left(n_{\mathrm{p}} / 1 \mathrm{~cm}^{-3}\right)^{-1} \mathrm{~s}$, and $t_{\pi \gamma} \sim 10^{18}\left(\mathrm{n}_{\mathrm{x}} / 1 \mathrm{~cm}^{-3}\right)^{-1} \mathrm{~s}$, respectively. Thus, charged pions would decay to $\mu$ and $\nu_{\mu}$ before interacting with the ambient photons and protons if $n_{\mathrm{x}} \leq 10^{21} \mathrm{~cm}^{-3}$ and $n_{\mathrm{p}} \leq 10^{17} \mathrm{~cm}^{-3}$. Finally, the production of neutrinos from the subsequent muon decay would proceed with high probability as long as the magnetic field does not exceed $B \approx 10^{6} \mathrm{G}$. This follows directly from the comparison of the decay time of muons, $t_{\mu}=\left(E_{\mu} / m_{\mu} c^{2}\right) \tau_{\mu} \simeq 0.2\left(E_{\mu} / 10 \mathrm{TeV}\right) \mathrm{s}$, with their synchrotron cooling time, $t_{\mathrm{sy}} \approx 0.07\left(B / 10^{6} \mathrm{G}\right)^{-2}\left(\mathrm{E}_{\mu} / 10 \mathrm{TeV}\right)^{-1} \mathrm{~s}$.

Thus, the ratio of $\gamma$-ray and neutrino production rates in a VHE source is expected to be of order of 1 or more, $\gamma / \nu \geq 1$. The absorption of $\gamma$-rays can, of course, significantly change the initial $\gamma / \nu$ ratio. While for galactic sources the external $\gamma$-ray absorption (due to interactions with the interstellar IR photon fields) is not dramatic up to several tens of $\mathrm{TeV}$ [24], in compact galactic objects like X-ray binaries the internal absorption can be huge. However, the absorption of gamma-rays does not imply that the information is lost; the secondary electrons initiate a cascade in the same radiation field and/or cool via synchrotron radiation. In the first case (which happens when the radiation density $w_{\mathrm{r}}$ exceeds the energy density of the magnetic field $B^{2} / 8 \pi$ ), the initial $\gamma$-rays are gradually reprocessed, down to energies at which the source becomes transparent. In binary systems containing luminous optical stars (like LS 5039) the main energy is released at $\mathrm{GeV}$ energies 25, 26, while in the case of particle acceleration in the accretion disk around a black hole (like Cygnus X-1), the initial energy is released mainly at $\mathrm{MeV}$ energies 27]. The cascade development in the photon field can be suppressed at the presence of a strong magnetic field, $B \geq \sqrt{8 \pi w_{\mathrm{r}}}$ (typically $10 \mathrm{G}$ or more). Interestingly, in this case the synchrotron radiation of pair-produced electrons extends well into the MeV gamma-ray energy band, and therefore its detection is not prevented ("screened") by the optical and X-ray backgrounds related to the optical star and the compact object. Thus, independent of the cooling regime (via synchrotron radiation and/or the Klein-Nishina cascades), the pair-produced electrons leave a detectable imprint in the form of $\mathrm{MeV}$ to $\mathrm{GeV}$ gamma-ray photons. So far the $\mathrm{MeV}$ and $\mathrm{GeV}$ $\gamma$-ray sky has been explored at the depth corresponding to the energy flux $\geq 10^{-10} \mathrm{erg} / \mathrm{cm}^{2} \mathrm{~s}$. Therefore the data available in the $\mathrm{MeV}-\mathrm{GeV}$ band from binary systems provide important, although not very restrictive upper limits on the energy flux of VHE neutrinos from these objects. With the arrival of AGILE and especially GLAST, the $\mathrm{MeV} / \mathrm{GeV} \gamma$-ray observations will play a more decisive role in predictions of VHE neutrinos from compact objects. 


\section{4. "Orphan" TeV neutrino sources?}

The multiwavelength approach to the estimates of VHE neutrino fluxes expected from potential cosmic accelerators indicate that the fluxes of the best-candidate persistent galactic neutrino sources cannot significantly exceed $\sim 10^{-11} \nu / \mathrm{cm}^{2} \mathrm{~s}$, i.e. most likely these sources are expected to be revealed at the level of statistically marginal signals. A possible exception could be "hidden" sources - proton accelerators completely shielded from us by a very thick $\left(\gg 100 \mathrm{~g} / \mathrm{cm}^{2}\right)$ gas material in which the energy of ultrarelativistic protons is converted with $100 \%$ efficiency to secondaries. At the same time, in such a thick shell of gas the high energy electromagnetic radiation would completely dissipate, and thus the source would becomes invisible because of much stronger background and forground thermal emission components. In such objects, the (highly unknown) total acceleration power of the source is the only model parameter that determines the neutrino flux; the source would be detectable by VHE neutrino detectors if the power of the "hidden" PeVatron exceeds, approximately by a factor of 10, the estimate given by Eq. (1).

There is another (more sophisticated) scenario of realization of "orphan" neutrino sources, i.e. objects with neutrino fluxes not accompanied by electromagnetic radiation. Such a possibility is related to the features of acceleration and radiation of particles in optically thick (with respect to the photon-photon pair production) relativistic flows which can be formed by hot plasma left behind a relativistic shock or exist in the form of jets. Generally, it is silently assumed that nonthermal particles and hence their radiation are isotropically distributed in the comoving frame. However, this assumption can easily be violated in relativistic shocks and jets with a strong impact on the emission properties, especially at very high energies. Namely, the beam pattern of relativistic jets with a bulk motion Lorentz factor $\Gamma$ in this energy domain appears much broader than the inverse Lorentz factor, $\Gamma^{-1}$. This results in an off-axis high energy emission [28] which is expected to be much brighter compared to the predictions derived from the standard Doppler boosting considerations applied to an isotropic (in the frame of the jet) source. However, in optically thick sources the electromagnetic radiation from super-critical particles is reprocessed through the electron-photon cascades, thus becomes isotropic in the jet frame, and, therefore, strongly collimated in the lab-frame. Consequently, the reprocessed electromagnetic radiation cannot be observable at large viewing angles. This effect does not concern the high energy neutrinos, therefore the jet, when viewed off-axis, may appear as an over-bright neutrino source with an arbitrarily large ratio of the neutrino luminosity to the total electromagnetic luminosity. It should be noted that acceleration of protons in relativistic shocks and shear flows can be significantly enhanced when it proceeds through the so-called converter mechanism 29]. This acceleration mechanism, which utilizes multiple conversions of protons to neutrons through photomeson reactions, has certain advantages compared to the standard diffusive shock acceleration scenario. It essentially diminishes particle losses downstream and provides penetration of particles deep into the upstream region allowing a highly desirable energy boost by a factor of $\Gamma^{2}$ at each shock encounter [29]. Since the copious neutrino production is an intrinsic feature of this scenario, the realization of the convertor acceleration mechanism in relativistic flows with large aspect angles would naturally lead to the appearance of an "orphan" VHE neutrino source.

\section{First galactic TeV neutrino sources to be detected...}

A possible hadronic origin of gamma-radiation of some of the $\mathrm{TeV} \gamma$-ray sources discovered by HESS in the galactic plane [31] makes them also potential emitters of high energy neutrinos (see e.g. ref.[30]). Recently, Vissani [11] Kistler and Beacom [12] and Kappes et al. [14] performed detailed calculations of the neutrino signal and background rates for the future $1 \mathrm{~km}^{3}$-volume scale neutrino telescopes based on the energy spectra and source-morphologies of galactic $\mathrm{TeV}$ $\gamma$-ray sources reported by HESS. The potential of the $\mathrm{km}^{3}$-volume class detectors is limitted, as 
mentioned above, by the detection of $\geq 1 \mathrm{TeV}$ neutrino fluxes at the level of $\geq 10^{-11} \nu / \mathrm{cm}^{2} \mathrm{~s}$ confined within an angle $\leq 1^{\circ}$. For power-law energy distributions of parent protons with powerlaw indices $\alpha=2-3$, the related $\gamma$-ray flux in the same energy interval is slightly, by a factor of 1 to 2 , higher [32, i.e. quite close to the gamma-ray flux of the Crab Nebula - the standard candle of $\mathrm{TeV} \gamma$-ray astronomy. Thus, the accompanied $\gamma$-ray flux in units of " 1 Crab" can be treated as the detection threshold of the galactic neutrino astronomy with $\mathrm{km}^{3}$-volume class instruments. Presently, in addition to the Crab Nebula itself, three more $\mathrm{TeV} \gamma$-ray sources are detected at the "1 Crab" level - two young shell-type SNRs RXJ 1713.7-3946 [33] and RXJ 0852.0-4622 [34] (Vela Jr), as well as a nearby PWN - Vela X [35].

\subsection{Shell type Supernova Remnants}

Young Supernova Remnants have been predicted, within a hadronic model, as extended TeV $\gamma$-ray and neutrino sources with shell type morphology and hard energy spectra extending to 100 $\mathrm{TeV}$ [36. The morphological and spectroscopic characteristics of young SNRs RXJ 1713.7-3946 and RXJ 0852.0-4622 reported by the HESS collaboration perfectly agree with these predictions, but ironically cannot yet be considered as an ultimate proof the hadronic model. The "trouble makers" are the alternative leptonic models which relate the $\mathrm{TeV} \gamma$-rays to inverse Compton scattering of electrons responsible also for the nonthermal X-ray emission. Although these models do not provide satisfactory explanations of the energy spectra of $\gamma$-rays, and require very low magnetic field of order of $10 \mu \mathrm{G}$ [33, 34], they unfortunately cannot be safely excluded. This prevent us from making a robust statement of detection of SNRs as sources of cosmic ray protons and nuclei. Although it is believed that detailed theoretical studies of SNRs in the context of their multiwavelength properties should allow us to arrive at certain conclusions concerning the origin of $\mathrm{TeV} \gamma$-ray emission, formally only the detection of $\mathrm{TeV}$ neutrinos from these objects can be considered as straightforward and unambiguous proof of acceleration of protons and nuclei. The predicted detection rates of $\geq 1 \mathrm{TeV}$ neutrinos (of order of a few events per one year) from the brightest $\gamma$-ray SNRs RAJ1713.7-3946 and RAJ0852.0-4622 by a km ${ }^{3}$-volume detector in the Mediterranean Sea, make the prospects of discovery of TeV neutrinos from these SNRs rather realistic. Unfortunately the locations of these two SNRs are not favorable for IceCube. The existence of SNRs of similar brightness in $\mathrm{TeV} \gamma$-rays located in the Northern Hemisphere will be explored, hopefully soon, by the VERITAS and MAGIC telescope systems. The search for $\mathrm{TeV} \gamma$-ray sources in the Cygnus region - one of the most prominent and promising parts of the galactic plane - is of special interest. The recent Milagro observations of this region revealed a diffuse $\gamma$-ray component with several hot spots [37, the strongest of which, MGRO J2019+37 could be a neutrino source with a flux close to the detection threshold of IceCube [38].

\subsection{Pulsar Wind Nebulae}

The close associations of some of the extended TeV galactic sources discovered by HESS with several well established synchrotron X-ray nebulae (MSH 15-52, PSR J1826-1334, Vela X, etc.) confirm the early theoretical predictions [39] on $\mathrm{TeV}$ gamma-ray visibility of young PWNe with spin-down "flux" $\left(L_{0} / 4 \pi d^{2}\right)$ exceeding $10^{34} \mathrm{erg} /\left(\mathrm{kpc}^{2} \mathrm{~s}\right)$. The broad-band spectral energy distributions of these sources are readily explained by the standard PWN model which assumes acceleration of ultrarelativistic electrons by the pulsar wind termination shock. Yet, in some of these systems particle acceleration could be driven by ions present in the relativistic pulsar wind [16]. These ions are expected to produce $\gamma$-rays and neutrinos via inelastic interactons with the ambient medium [40, 41, 42, 43]. In this regard, the extended TeV source associated with the pulsar PSR B0833-45 (Vela X) is a possible candidate for such a "hadronic plerion". Indeed, although the observed $\gamma$-ray emission can be interpreted as inverse Compton emission of nonthermal electrons [35, one needs to make some strong (non-trivial) assumptions in order to explain the rather unusual spectrum of this source with photon index $\Gamma \simeq 1.5$ and exponential 
cutoff around $14 \mathrm{TeV}$. The steady-state electron distribution constrained by $\gamma$-ray data requires an $E^{-2}$ type power-law spectrum with a sharp cutoff around $70 \mathrm{TeV}$. Such a spectrum of electrons can be interpreted only in terms of negligible synchrotron cooling, which would be possible only in the case of unusually low nebular magnetic field (a few $\mu \mathrm{G}$ or less). Moreover, the total energy in relativistic electrons and in the magnetic field, which is required to match the observed Xray and gamma-ray fluxes, is only a negligible fraction $(\approx 0.1 \%)$ of the pulsar spin-down energy released over the pulsar's life-time $1.1 \times 10^{4} \mathrm{yr}$. This begs the question as to where the remaining energy has gone? Interestingly if we assume that a large fraction of the spin-down luminosity of the pulsar is carried out by relativistic protons and nuclei, one can satisfactorily explain both the absolute flux and the spectrum of $\mathrm{TeV} \gamma$-rays of this unusual source [44. Remarkably, the $\mathrm{TeV}$ neutrino flux expected within this scenario should be detectable by KM3NeT 14. This makes the Vela $\mathrm{X}$ as one of the best-bet candidates to be the first detected astronomical $\mathrm{TeV}$ neutrino source. For the IceCube detector an obvious target representing this source population is the Crab Nebula.

\subsection{Compact Binary Systems}

The recent detections of $\mathrm{TeV} \gamma$-rays from two binary systems tentatively called microquasars - LS 5039 by HESS [45] and LSI 61303 by MAGIC [46] - are amongst the most exciting discoveries of observational gamma-ray astronomy in the very high energy regime. This result clearly demonstrates that the galactic binary systems containing a luminous optical star and a compact object (a black hole or a pulsar/neutron star), are sites of effective acceleration of particles (electrons and/or protons) to multi-TeV energies. As usual, whether the $\gamma$-rays are of hadronic or leptonic origin is a key question which however does not have a straightforward answer (see e.g. ref. 47). The critical analysis of conditions of particle acceleration and radiation in these sources, based on the temporal and spectral behavior of $\mathrm{TeV} \gamma$-ray emission, in particular on the modulation of the TeV flux of LS 5039 with a period of 3.9 day, and the extension of its energy spectrum to $10 \mathrm{TeV}$ and beyond, reduces the possible interpretations to a few options. One of them gives a preference to the hadronic origin of $\mathrm{TeV}$ photons, especially if they are produced within the binary system [25]. If so, the detected $\gamma$-rays should be accompanied by a flux of high energy neutrinos emerging from the decays of $\pi^{ \pm}$mesons produced by $p$ - $p$ and/or $p \gamma$ interactions. The neutrino fluxes, which can be estimated on the basis of the detected $\mathrm{TeV}$ $\gamma$-ray fluxes, taking into account the severe internal $\gamma \gamma \rightarrow e^{+} e^{-}$absorption, depend significantly on the location of $\gamma$-ray production region(s) [48, 49]. The minimum neutrino flux above $1 \mathrm{TeV}$ is expected to be at the level of $10^{-12} \nu / \mathrm{cm}^{2} \mathrm{~s}$; however, it could be much higher - by a factor of 10 , or even more. The detectability of the $\mathrm{TeV}$ neutrino signals significantly depends on the high energy cutoff in the spectrum of parent protons. If the spectrum of accelerated protons continues to $100 \mathrm{TeV}$ and beyond, the predicted neutrino fluxes of LS 5039 and LSI 61303 can be probed by KM3NeT [25] and IceCube [50, 51] high energy neutrino detectors.

\section{References}

[1] Halzen F 2007 Science $\mathbf{3 1 5} 66$

[2] Katz U F 2006 NIMPA $\mathbf{5 6 7} 457$

[3] Lipari P 2006 NIMPA $\mathbf{5 6 7} 405$

[4] Waxman E 2007 Science $\mathbf{3 1 5} 63$

[5] Rachen J P and Mészäros P 1998 Phys. Rev. D 58 id. 123005

[6] Mészäros 2006 Rep. Prog. Phys. 692259

[7] Dermer C D and Atoyan A 2006 New Journal of Physics 8122

[8] HESS collaboration 2006 Astrophys $J \mathbf{6 3 6} 777$

[9] Atkins R et al. (Milagro collaboration) 2005 Phys. Rev. Lett. 95251103

[10] McKay T A et al. (CASA-MIA collaboration) 1993 Astrophys. J 417742

[11] Vissani F 2006 Astropart. Physics 26310 
[12] Kistler M and Beacom J F Phys. Rev. D 74 id. 063007

[13] Distefano C 2007 Astrophys Space Sci, in press, astro-ph/0608514

[14] Kappes A, Hinton J, Stegmann Ch and Aharonian F A 2007 Astrophys. J. 656870

[15] Malkov M A and Drury L 2001 Report on Progress in Physics 64429

[16] Arons J 1995 Space Sci. Rev. 75235

[17] Berezinsky V S 1977, in Proc. "Ultra High Energy Neutrinos and Detection Possibilities by DUMAND" (FERMILAB, Batavia) 229

[18] Vestrand W T and Eichler D 1982 Astrophys. J. 261251

[19] Hillas A M 1984 Nature 31250

[20] Gaisser T K and Stanev T 1985 Phys. Rev. Lett. 542265

[21] Anchordoqui L A, Torres D F, McCauley T P, Romero G E and Aharonian F A 2003 Astrophys. J. 589481

[22] Levinson A and Waxman E 2001 Phys. Rev. Lett. 87 id. 171101

[23] Distefano C, Guetta D, Waxman E and Levinson A 2002 Astrophys. J. 575378

[24] Moskalenko I V, Porter T A and Strong A W 2006 Astrophys. J. 640 L155

[25] Aharonian F, Anchordoqui L, Khangulyan D and Montaruli T 2006J of Physics: Conf. Ser. 39408

[26] Bednarek W 2006 MNRAS 368579

[27] Aharonian F A and Vardanian V V 1985 Astrophys. Space Sci. 11531

[28] Derishev E V, Aharonian F A and Kocharovsky, Vl V 2007 Astrophys. J. 655980

[29] Derishev E V, Aharonian F A, Kocharovsky, V V and Kocharovsky, Vl V 2003 Phys. Rev. D 68 id.043003

[30] Dermer C D 2007 Proc. of TeV-Particle Astrophysics II, in press

[31] Aharonian F A 2007 Science 31570

[32] Kelner S R, Aharonian F A and Bugayov V V 2006 Phys. Rev. D 74 id.034018

[33] HESS collaboration 2004 Nature 432 75; 2006 Astron. Astrophys. 449223

[34] HESS collaboration 2005 Astron. Astrophys. 437 L7

[35] HESS collaboration 2006 Astron. Astrophys. 448 L43

[36] Drury L, Aharonian F A and Völk H J 1994 Astron. Astrophys 287959

[37] Abdo A A et al (Milagro collaboration) 2006, Astrophys. J., submitted, astro-ph/0611691

[38] Beacom J F, Kistler M D 2007 Phys Rev. D (submitted), astro-ph/0701751

[39] Aharonian F A, Atoyan A M and Kifune T 1997 MNRAS 291162

[40] Atoyan A M and Aharonian F A 1996 MNRAS 278525

[41] Bednarek W and Protheroe R J 1997 Phys. Rev. Lett. 792616

[42] Bednarek W 2003 Astron. Astrophys 4071

[43] Amato E, Guetta D and Blasi P 2003 Astron. Astrophys. 402827

[44] Horns D, Aharonian F, Santangelo A, Hoffmann A and Masterson C 2006 Astron. Astrophys 451 L51

[45] HESS collaboration 2005 Science 309 746; 2006 Astron. Astrophys 460743

[46] Albert J et al (MAGIC collaboration) 2006 Science 3121771

[47] Paredes J M 2007, in "Frontier Objects in Astrophysics and Particle Physics"; astro-ph/0609168

[48] Dubus G 2006 Astron. Astrophys. 4519

[49] Boettcher M 2007 Astropart. Physics., in press: astro-ph/0609136

[50] Christiansen H R, Orellana M and Romero G E 2006 Phys. Rev. D 73063012

[51] Torres D F and Halzen Francis 2006 astro-ph/0607368 